Artículo

\title{
Diseño universal para el aprendizaje como metodología docente para atender a la diversidad en la universidad
}

\author{
Emiliano Díez Villoria ${ }^{a}$ y Sergio Sánchez Fuentes ${ }^{\mathrm{b}, *}$ \\ a Departamento de Psicología Básica, Psicobiología y Metodologías de las Ciencias del Comportamiento, Instituto Universitario de Integración en la Comunidad (INICO), \\ Universidad de Salamanca, Salamanca, España \\ ${ }^{\mathrm{b}}$ Departamento de Educación, Universidad Católica de Murcia, Murcia, España
}

\section{INFORMACIÓN DEL ARTÍCULO}

\section{Historia del artículo:}

Recibido el 23 de octubre de 2014

Aceptado el 30 de diciembre de 2014

On-line el 20 de febrero de 2015

\section{Palabras clave:}

Formación del profesorado

Universidad

Diseño Universal para el Aprendizaje

Atención a la diversidad

Discapacidad

\section{R E S U M E N}

Este artículo aborda la necesidad de formación del profesorado en metodologías que atiendan a la diversidad en la universidad. Por un lado, se defiende la obligación legal de asegurar la igualdad de oportunidades para todos los estudiantes. Por otro lado, también se plantea que una de las vías de mejora debe ser la formación del profesorado en paradigmas de referencia creados para atender a la diversidad de estudiantes, especialmente, el diseño universal para el aprendizaje. También se revisan los resultados de un estudio en el que las demandas de adaptaciones curriculares de estudiantes universitarios con discapacidad se ponen en correspondencia con los principios y pautas del diseño universal para el aprendizaje. Los resultados permiten aventurar que el diseño curricular bajo los principios del diseño universal para el aprendizaje podría minimizar las demandas de adaptaciones especiales, incluso en grupos de estudiantes con mayores dificultades. Por último, se revisan algunas experiencias de formación del profesorado en diseño universal para el aprendizaje y se describe una experiencia formativa específica puesta en marcha en un plan de formación de personal docente universitario. Con todo ello, pretendemos acercar a la comunidad universitaria nuevos modos de afrontar el reto de diseñar currículos inclusivos que tengan en cuenta a todos los estudiantes.

(C) 2014 Instituto de Ciencias de la Educación de la Universidad de Oviedo. Publicado por Elsevier España, S.L.U. Este es un artículo Open Access bajo la licencia CC BY-NC-ND

(http://creativecommons.org/licenses/by-nc-nd/4.0/).

\section{Universal design for learning as a teaching method in order to meet the need for diversity in universities}

\begin{abstract}
A B S T R A C T
This paper looks at how diversity is dealt with in university from a teacher training perspective. On the one hand, it is a legal obligation of universities to ensure equal opportunities for all students. On the other hand, it is argued that teacher training in paradigms and frameworks created to deal with diversity are also a way of improving. In this context, a universal design for learning paradigm will be presented, describing some research outcomes on its application. The article also reviews the results of an investigation into the demands by students with disability for adaptations to the curriculum, and their relationship with the universal design for learning principles. These results suggest that curriculum design following the principles of universal design for learning could minimize the demands for curriculum adaptations, even for students groups that experience more difficulties. Lastly, some experiences of teacher training with the universal design for learning will be reviewed, as well as the specific experience in implementing a
\end{abstract}

\footnotetext{
* Autor para correspondencia: Universidad Católica de Murcia, Campus de los Jerónimos, N. 135 Guadalupe, 30107 (Murcia). Tel.: +968 278801.

Correo electrónico: ssanchez@ucam.edu (S. Sánchez Fuentes).
} 
plan for university teacher training. With all this, the intention is to bring new ways to meet the challenge in the design of inclusive curricula for ALL students to the university community.

(c) 2014 Instituto de Ciencias de la Educación de la Universidad de Oviedo. Published by Elsevier España, S.L.U. This is an open access article under the CC BY-NC-ND license (http://creativecommons.org/licenses/by-nc-nd/4.0/).

\section{La atención a la diversidad en la universidad}

En nuestra sociedad, los derechos fundamentales de todas las personas pasan por el acceso a una serie de servicios básicos que garantizan la realización personal como seres humanos. Uno de estos derechos es el acceso a una educación de calidad que garantice que todas las personas tienen las mismas oportunidades de aprender y, por tanto, de formarse como ciudadanos. Por ello, la educación debería considerar la atención a la diversidad en las aulas como uno de sus pilares básicos; entendiendo como atención a la diversidad, la puesta en marcha de acciones que tengan como objetivo que todas las personas, independientemente de cuáles sean sus características, puedan acceder en igualdad de oportunidades al proceso educativo.

La atención a la diversidad en la universidad está fundamentada desde la propia legislación universitaria. Esta necesidad parte del Real Decreto 1393/2007, de 29 de octubre, por el que se establece la ordenación de las enseñanzas universitarias oficiales, que en su Principio 5, en el cual se describen los principios generales que deberán inspirar el diseño de los títulos universitarios, se describe cómo la creación de currículos académicos se debe realizar desde el respeto y promoción de los Derechos Humanos y los principios de accesibilidad universal y diseño para todos; que a su vez, deriva de lo expuesto en la disposición final décima de la Ley 51/2003, de 2 de diciembre, de Igualdad de oportunidades, no discriminación y accesibilidad universal de las personas con discapacidad, como ley fundamental que rige los derechos de las personas con discapacidad. Incluso desde la propia Ley Orgánica de Universidades 4/2007, en concreto desde la disposición adicional vigésimo cuarta, se describe cómo debe ser la inclusión de las personas con discapacidad en las universidades. Y es que la LOU establece para la educación universitaria, entre otros principios, el principio de no discriminación e igualdad de oportunidades a través de medidas de acción positiva. Ejemplos de estas medidas son, por ejemplo, la exención de tasas y precios públicos para la obtención de un título universitario para las personas con diversidad funcional, ayudas al transporte, programas de deporte adaptado, tutorías, apoyo institucional o prioridad en la matrícula. Además, otras legislaciones relacionadas con la universidad como el Real Decreto 1791/2010, de 30 de diciembre, por el que se aprueba el Estatuto del Estudiante Universitario o el Real Decreto 1892/2008, de 14 de noviembre, por el que se regulan las condiciones para el acceso a las enseñanzas universitarias oficiales, mantienen esta filosofía en sus textos haciendo que la atención a la diversidad en la universidad española esté claramente regulada para su puesta en funcionamiento. Y de hecho, todas estas medidas han provocado que el colectivo de estudiantes con diversidad funcional haya aumentado a lo largo de la última década, pasando de los casi 8.000 estudiantes en el curso académico 2004-2005 (Peralta, 2007) hasta cerca los 20.000 estudiantes con discapacidad que se estima se encontraban matriculados en el curso 2012-2013 (Fundación Universia, 2013).

Así, teniendo en cuenta la legislación universitaria en materia de atención a la diversidad, así como la necesidad de que el diseño de los títulos universitarios se inspire en concepciones basadas en accesibilidad universal y diseño para todos, parece clara la necesidad de que el profesorado universitario adquiera competencias sobre diseño curricular basado en el diseño universal para el aprendizaje. El trabajo que presentamos se ubica en este contexto. En primer lugar, introduciremos el paradigma del diseño universal para el aprendizaje y presentaremos los resultados de una investigación sobre las necesidades de medidas curriculares percibidas por parte de estudiantes con discapacidad universitarios; creemos que los resultados de este estudio, aunque derivados de una muestra limitada, servirán como ejemplo y justificación de la necesidad de abordar medidas basadas en el diseño universal en el diseño curricular universitario. En segundo lugar, revisaremos algunas experiencias de enseñanza de diseño universal a profesores y presentaremos la experiencia llevada a cabo en el programa de formación del profesorado de la Universidad de Salamanca sobre diseño universal para el aprendizaje. Todo ello nos permitirá concluir que la formación del profesorado en estos nuevos paradigmas proactivos de atención a la diversidad se configura, sin duda, como un elemento esencial para acercarnos al objetivo de ofrecer una educación universitaria en igualdad de condiciones para todos los estudiantes.

\section{El diseño universal para el aprendizaje en la educación superior}

El diseño universal o diseño para todos, entendido como la adopción de concepciones abiertas e inclusivas respecto al modo de diseñar dispositivos y servicios es uno de los aspectos claves en el incremento en la participación de la personas con discapacidad en la universidad (Sánchez, Díez, Verdugo, Iglesias y Calvo, 2011). Como bien señalan McGuire, Scott y Shaw: «el diseño universal aplicado a la educación puede ser un nuevo paradigma que permita hacer efectiva la implementación de la inclusión y proporcionar acceso a la educación general del currículo» (2006, p. 167).

Una de las principales vías que defiende el diseño universal para lograr este objetivo es la creación de currículos accesibles que contemplen la diversidad de estudiantes dentro de las aulas; y es que, este aspecto debería ser prioritario a la hora de reflexionar sobre los procesos de diseño curricular (Díez, Campo y Sánchez, 2011; Díez et al., 2011; Sánchez et al., 2011). Un currículo planificado bajo los principios del diseño universal está específicamente diseñado para atender a un conjunto diverso de estudiantes con un amplio rango de habilidades sensoriales, motrices, cognitivas, afectivas y lingüísticas (McGuire, Scott y Shaw, 2006; Hitchcock y Stahl, 2003). Pero no solo los estudiantes con necesidades específicas pueden beneficiarse, sino que «todos los miembros de la comunidad universitaria podrían verse beneficiados por la aplicación de los principios del diseño universal a las experiencias de enseñanza y aprendizaje» (Darr y Jones, 2008, p.107).

La aplicación del paradigma del diseño universal al ámbito educativo universitario se pone de manifiesto a través de diversos enfoques que, aun manteniendo un eje vertebrador común, difieren en algunos aspectos específicos. Un trabajo llevado a cabo por Ruiz, Solé, Echeita, Sala, y Datsira (2012), describe un análisis pormenorizado del desarrollo del concepto del diseño universal aplicado en la enseñanza, y más particularmente, en la enseñanza superior.

Fundamentalmente, se podría hablar de 4 concepciones distintas a la hora de aplicar el paradigma del diseño universal a la educación superior. En primer lugar citaremos una de las concepciones con mayor difusión, el diseño universal para el aprendizaje (DUA), descrito desde el Center for Applied Special Techonology. 
El DUA es un marco conceptual que aborda el principal obstáculo de los entornos educativos: los currículos inflexibles que plantean barreras no intencionadas al aprendizaje (CAST, 2011). Desde esta perspectiva, aquellos estudiantes que no se adaptan a una "normalidad" preestablecida son los que tienen más posibilidades de padecer una segregación en su proceso de enseñanza y aprendizaje, debido a sus características y condiciones personales, que no se tienen en cuenta dentro de lo que se podría considerar como un falso promedio. Tanto estos estudiantes como aquellos que se pueden considerar dentro del promedio podrían no tener satisfechas sus necesidades de aprendizaje debido a los diseños curriculares inadecuados (National Center for Universal Design for Learning, 2012). Por ejemplo, si los contenidos digitales obligatorios de una asignatura no se ofrecen en formatos accesibles, esto podría provocar un problema para estudiantes que requieran el acceso a la información mediante dispositivos especiales o adaptados (e.g., un estudiante con discapacidad visual que utilice un lector de pantalla).

El DUA defiende que los currículos diseñados bajo los principios del diseño universal son la clave para la atención a la diversidad de los estudiantes. Y plantea que el diseño se debe sustentar en tres principios fundamentales derivados de numerosos estudios empíricos y en especial de investigaciones del ámbito de las neurociencias (Meyer y Rose, 2005, 2006, 2009; Rose y Meyer, 2000, 2002). Estos tres principios son: a) proporcionar múltiples formas de representación (el «qué» del aprendizaje); b) proporcionar múltiples formas para la acción y la expresión (el «cómo» del aprendizaje); y c) proporcionar múltiples formas de participación (el "porqué» del aprendizaje). Cada principio, a su vez, se divide en tres pautas concretas en las que se especifica con más detalle distintos medios para seguir esos principios.

Otro de los enfoques de aplicación del diseño universal a la educación universitario es el enfoque del diseño instruccional universal (DIU), que, en resumen, plantea la aplicación de los siete principios del diseño universal arquitectónico al ámbito pedagógico. En concreto, Bryson (2003) lo define como: «el incremento de las capacidades instruccionales en entornos universitarios, a través de la potenciación y el fomento de las competencias y los conocimientos del profesorado» (p. 39).

Y en esta misma línea también destaca el enfoque del diseño universal para la instrucción, desde el que Scott, McGuire y Shaw (2001) proponen la aplicación de los 7 principios del diseño universal originales y añaden dos principios más, relacionados con aspectos importantes para la educación: a) las comunidades de aprendizaje y b) el clima educativo instruccional.

Por último, podríamos citar el enfoque de diseño universal en educación, aunque no se puede considerar como un paradigma en sí mismo, ni de la misma envergadura que los otros enfoques que acabamos de describir Ruiz et al. (2012). La diferencia fundamental entre el diseño universal en educación y el diseño universal para el aprendizaje sería la utilización de la palabra educación, que implica una concepción más amplia que aprendizaje en cuanto al alcance de los procesos y situaciones a los que se refiere. Además el diseño universal en educación se centra en el contexto universitario.

Con el objetivo de obtener evidencia empírica que avale la aplicación del paradigma del diseño universal al ámbito educativo universitario, es necesario desarrollar investigaciones que demuestren a la comunidad educativa cómo las actuaciones basadas en la aplicación de los principios del diseño universal a la educación trabajan a favor de la inclusión de todos los estudiantes (Spooner, Baker, Harris, Ahlgrim-Delzell y Browder, 2007).

Dada la juventud de estos enfoques, no existen aún demasiados estudios en los que se analice su utilidad en el ámbito universitario. Pero ya se pueden citar algunos estudios relevantes. Un ejemplo es el trabajo de Rose, Harbour, Johnston, Daley y Abarbanell (2006), en el que describen la implementación de un curso denominado «El
Reto de las Diferencias Individuales» en la Universidad de Harvard (Estados Unidos) siguiendo los principios del DUA. Como característica fundamental de la realización de este seminario, habría que destacar la cantidad de recursos que se pusieron a disposición de los estudiantes que formaban parte del mismo. Entre los recursos más interesantes podríamos destacar: un intérprete de lengua de signos para estudiantes sordos; texto alternativo en cualquier imagen presentada a lo largo de la duración del curso para personas con baja visión; y grabación en vídeo de las lecciones magistrales y su posterior alojamiento en un página Web creada específicamente para el desarrollo del curso - lo que permitía visualizar cualquier parte de las conferencia en el momento deseado después de la lección presencial-. Los autores del trabajo concluyen que la deficiencia en el aprendizaje está centrada en el ambiente y no en los estudiantes (Rose et al., 2006) y que el diseño universal para el aprendizaje puede beneficiar a los estudiantes con algún tipo de discapacidad, pero que, en general, la adopción de estrategias basadas en DUA favorece a todos los estudiantes.

Otro ejemplo es la experiencia llevada a cabo por Yuval, Procter, Korabik y Palmer (2004) en la Universidad de Guelph. Estos investigadores iniciaron la puesta en marcha de una serie de acciones en 9 asignaturas de dicha universidad, que fueron modificadas siguiendo los principios del diseño universal en el ámbito educativo universitario. Los objetivos principales del programa fueron fundamentalmente dos: a) estimular a los profesores en la aplicación de los principios del diseño universal aplicado a la educación en las asignaturas del proyecto y b) evaluar el impacto de la aplicación de estos principios. Las asignaturas seleccionadas en el proyecto fueron rediseñadas bajo la óptica del diseño universal. Para ello, en algunos casos se modificaron los currículos por completo y, en otros, se introdujeron mejoras basadas en el diseño universal. La evaluación incluyó cuestionarios a los estudiantes, observaciones en clase y entrevistas, tanto a profesores como a estudiantes de las asignaturas implicadas. El objetivo fundamental de dicha evaluación fue analizar el grado de aplicación de las medidas basadas en el diseño universal y comprobar si la aplicación de dichas medidas tenía una influencia sobre la experiencia educativa de los estudiantes.

En primer lugar, los resultados mostraron una relación positiva entre el nivel de implementación de medidas de diseño universal y la autoeficacia académica de los estudiantes en aspectos como la concentración en clase, la memorización, la comprensión, la explicación y discriminación de conceptos o la toma de apuntes. En segundo lugar, los resultados demostraron que los estudiantes de asignaturas que implementaron más medidas de diseño universal manifestaban más emociones positivas y menos negativas que los de asignaturas con menos medidas implementadas. Igualmente importante fue la evaluación proporcionada por los profesores de las asignaturas quienes señalaron una mejora de sus conocimientos acerca del diseño universal aplicado a la educación superior, así como un mayor compromiso con este paradigma para sus futuras actividades académicas.

De esta breve revisión se puede concluir, en primer lugar, que en la última década han surgido suficientes enfoques y marcos de referencia que pueden facilitar y mostrar el camino a seguir para dar respuesta a los requisitos planteados por los recientes desarrollos legislativos en relación a la atención a la diversidad en el ámbito universitario. En segundo lugar, los resultados de las escasas investigaciones en las que se ha puesto a prueba la utilidad del paradigma del diseño universal para el aprendizaje, señalan resultados positivos para profesores y estudiantes por la aplicación de sus principios.

Por lo tanto, el objetivo de este trabajo, en términos generales, es analizar si el diseño universal para el aprendizaje sirve como metodología docente para dar respuesta a la diversidad en el ámbito universitario. 


\section{¿Es el diseño universal para el aprendizaje la respuesta para la atención a la diversidad?: percepción de estudiantes universitarios con discapacidad sobre las necesidades de adaptaciones curriculares}

Ante la necesidad de disponer de experiencias que avalen la aplicación de los principios del diseño universal para el aprendizaje en la educación universitaria, a continuación presentamos un estudio descriptivo en el que se analizaron las demandas sobre adaptaciones curriculares de estudiantes con discapacidad bajo la óptica del diseño universal para el aprendizaje. Los estudiantes universitarios con discapacidad son uno de los colectivos que mayores dificultades encuentran en su paso por la universidad. Por ello, el análisis de la correspondencia entre sus demandas y los principios del diseño universal para el aprendizaje puede constituir un argumento importante a la hora de justificar y avalar la implantación de medidas relacionadas con la promoción del DUA en la educación universitaria. Y por supuesto, también contribuirá a la definición de aquellas acciones de diseño curricular que se deben conocer por parte de los docentes para poder programar una enseñanza universitaria de calidad que asegure la igualdad de oportunidades para todos.

\section{Participantes}

La población que participó en este trabajo estaba formada por un grupo de estudiantes con discapacidad de las universidades españolas. La muestra fue seleccionada mediante un muestreo no probabilístico de conveniencia, contactando con servicios de atención a estudiantes con diversidad funcional de 74 universidades españolas que proporcionaron contacto con un total de 98 estudiantes.

Los estudiantes pertenecían a 10 universidades españolas distintas. Más de la mitad estaban matriculados en titulaciones de la rama de las Ciencias Sociales, y el resto se repartían proporcionalmente en las otras ramas de conocimiento. En cuanto al tipo de discapacidad, el $40 \%$ de los estudiantes eran personas con discapacidad física, en un 23,33\% eran personas con algún tipo de dificultad visual y, en menor medida, los estudiantes reportaron otro tipo de discapacidades (sensorial auditiva, enfermedad crónica o enfermedad mental).

\section{Procedimiento}

Todos los estudiantes cumplimentaron un cuestionario en formato papel sobre adaptaciones curriculares traducido y adaptado de un estudio previo (Hill, 1996). En el cuestionario se solicitaba a los estudiantes que valorasen su necesidad respecto a adaptaciones curriculares y su percepción sobre el grado de predisposición de los profesores para llevarlas a cabo. El cuestionario fue aplicado por profesionales entrenados para tal propósito. Se llevó a cabo in situ en las diferentes universidades participantes en el estudio.

Aunque los resultados del cuestionario ya han sido objeto de análisis en otro trabajo (Moral, 2009) el interés en este estudio se centró en el análisis de las respuestas de carácter abierto que los estudiantes realizaron en cada apartado señalando cualquier adaptación que consideraban necesaria en tres ámbitos cubiertos por el cuestionario: a) las clases; b) las tareas y trabajos; y, c) los exámenes y las evaluaciones. En concreto, el análisis consistió en clasificar las respuestas atendiendo al principio o principios del diseño universal para el aprendizaje al que hacían referencia. La clasificación de las respuestas en cada una de las pautas se llevó a cabo por los autores de manera consensuada. Este procedimiento se fundamenta en el amplio conocimiento del documento de las Pautas 2.0 sobre el DUA, ya que estas han sido traducidas al castellano para otro trabajo (Sánchez et al., 2011) por parte de los autores.

Así, todas las respuestas de los estudiantes se categorizaron en alguna o algunas de las nueve pautas que conforman los tres principios del diseño universal para el aprendizaje (National Center for Universal Design for Learning, 2012).

Las Pautas 2.0 sobre el Diseño Universal para el Aprendizaje, son un documento en el que se describen para cada uno de los tres principios del DUA, una serie de pautas y puntos de verificación que sustentan la puesta en práctica del DUA. En resumen, las pautas están organizadas de lo más general (Principio) hasta lo más específico (Punto de verificación). Y, en cada principio, las pautas siguen una organización jerárquica vertical, desde lo más sencillo en términos de procesamiento cognitivo y de facilidad de implementación (e.g., accesibilidad de contenidos, variación en modos de respuesta o captación inicial del interés) hasta lo más complejo (e.g., recursos dirigidos a mejorar la comprensión, las funciones ejecutivas o la implicación activa en el proceso de aprendizaje).

\section{Resultados: relación entre las demandas de los estudiantes con discapacidad y los principios del diseño universal para el aprendizaje}

Los estudiantes señalaron un total de 63 adaptaciones curriculares necesarias adicionales a las citadas originalmente en el cuestionario. En la siguiente tabla se presenta el número de respuestas distintas en función de la pauta en la que fueron clasificadas.

En primer lugar cabe destacar que todas las adaptaciones o medidas demandadas por los estudiantes con discapacidad pudieron ser clasificadas en alguno de los principios y pautas del DUA. En ningún momento se comentó a los estudiantes ningún aspecto relacionado con el paradigma del DUA y mediante sus respuestas simplemente estaban describiendo aquellas actuaciones que, bajo su conocimiento, podrían mejorar su experiencia educativa en la universidad. En este sentido, parece razonable pensar que, si uno de los principales colectivos que se vería beneficiado de actuaciones de atención a la diversidad expresa medidas que se relacionan claramente con el DUA, este es un paradigma que se debe tener en cuenta en la formación de profesorado universitario como instrumento para lograr una mejor atención a la diversidad (tablas $1-3)$.

En resumen, las respuestas de los estudiantes tenían que ver con temas que el DUA propone como medidas para la creación de

Tabla 1

Principios y pautas del Diseño Universal para el Aprendizaje

\begin{tabular}{|c|c|c|}
\hline $\begin{array}{l}\text { Principio I. Proporcionar múltiples formas de } \\
\text { representación (el «qué» del aprendizaje) }\end{array}$ & $\begin{array}{l}\text { Principio II. Proporcionar múltiples formas para la } \\
\text { acción y la expresión (el «cómo» del aprendizaje) }\end{array}$ & $\begin{array}{l}\text { Principio III. Proporcionar múltiples formas de } \\
\text { participación* (el «porqué» del aprendizaje) }\end{array}$ \\
\hline \multicolumn{3}{|l|}{ Pautas } \\
\hline 1. Proporcionar opciones para la percepción & 4. Proporcionar opciones para la interacción física & 7. Proporcionar opciones para captar el interés \\
\hline $\begin{array}{l}\text { 2. Proporcionar múltiples opciones para el lenguaje, } \\
\text { los símbolos y las expresiones matemáticas }\end{array}$ & $\begin{array}{l}\text { 5. Proporcionar opciones para la expresión y la } \\
\text { comunicación }\end{array}$ & $\begin{array}{l}\text { 8. Proporcionar opciones para mantener el } \\
\text { esfuerzo y la persistencia }\end{array}$ \\
\hline 3. Proporcionar opciones para la comprensión & $\begin{array}{l}\text { 6. Proporcionar opciones para las funciones } \\
\text { ejecutivas }\end{array}$ & 9. Proporcionar opciones para la autorregulación \\
\hline
\end{tabular}

Fuente: adaptada de NCUDL, 2012. 
Tabla 2

Número de adaptaciones demandadas en función de la pauta del diseño universal para el aprendizaje con la que se corresponde

\begin{tabular}{lc}
\hline Pauta & $\begin{array}{c}\text { Número de } \\
\text { respuestas }\end{array}$ \\
\hline $\begin{array}{l}\text { Pauta 1. Proporcionar diferentes opciones para la } \\
\quad \text { percepción }\end{array}$ & 15 \\
$\begin{array}{l}\text { Pauta 2. Proporcionar múltiples opciones para el lenguaje } \\
\quad \text { y los símbolos }\end{array}$ & 5 \\
Pauta 3. Proporcionar opciones para la compresión & 16 \\
Pauta 4. Proporcionar múltiples medios físicos de acción & 5 \\
Pauta 5. Proporcionar opciones para la expresión y la & \\
$\quad$ fluidez de la comunicación & 0 \\
Pauta 6. Proporcionar opciones para las funciones & \\
$\quad$ ejecutivas & 12 \\
Pauta 7. Proporcionar opciones para captar el interés & 0 \\
Pauta 8. Proporcionar opciones para mantener el esfuerzo & \\
$\quad$ y la persistencia & 6 \\
Pauta 9. Proporcionar opciones para la autorregulación & 6 \\
\hline
\end{tabular}

Fuente: elaboración propia.

currículos inclusivos. Por ejemplo, los comentarios clasificados en las pautas del Principio 1 tenían que ver con las adaptaciones de los materiales tanto en su formato (e.g., formatos accesibles) como en los apoyos ofrecidos a los estudiantes para su asimilación. Los comentarios clasificados en las pautas del Principio 2 tenían que ver con asuntos como la accesibilidad de las instalaciones y los dispositivos o la flexibilidad en los formatos de respuesta y evaluación. Y los comentarios de las pautas del Principio 3 tenían que ver con la captación del interés mediante la minimización de distracciones o la variedad de actividades y con la necesidad de formación y concienciación de los profesores universitarios en aspectos relacionados con la discapacidad y su atención.

A modo de ejemplo, y para ilustrar el tipo de medidas demandadas por los estudiantes a continuación se presentan algunas respuestas clasificadas en sus respectivas pautas.

Aunque derivados de una muestra limitada, los resultados obtenidos indican que las demandas de adaptaciones curriculares de los estudiantes con discapacidad están relacionadas con los principios del diseño universal para el aprendizaje y, por tanto, la necesidad de estas adaptaciones "especiales» podría reducirse diseñando los currículos bajo los principios del DUA. Por ejemplo, si un contenido o actividad se ofrece en formatos accesibles y con flexibilidad en los modos de respuesta demandados al estudiante no sería necesario realizar una adaptación específica para grupos concretos de estudiantes. Es más, este tipo de medidas beneficiarían a todos los estudiantes, ya que los formatos accesibles, por seguir con el ejemplo, benefician tanto al estudiante con discapacidad visual que consume los contenidos mediante un lector de pantalla como al estudiante que quiere disponer de los contenidos en su dispositivo móvil para echarles un vistazo mientras llega a la facultad en el transporte público o al que prefiere transformar el texto a voz y escucharlo mientras hace ejercicio.

Teniendo en cuenta la importancia de este tipo de medidas, consideramos adecuado proponer que, a través de los programas de formación del profesorado universitario o por el medio que sea, se creen contenidos y se proporcionen las competencias necesarias para que los docentes puedan llevar a cabo su actividad académica atendiendo a las necesidades de todos los estudiantes mediante la aplicación de los principios del DUA.

\section{¿Cómo integrar el diseño universal para el aprendizaje en la formación del profesorado?}

Además de las experiencias globales de la aplicación del DUA a la educación universitaria citadas anteriormente, consideramos de especial relevancia aquellos estudios que se han centrado en analizar cómo la formación del profesorado universitario en conocimientos y técnicas sobre la aplicación del DUA en el diseño de currículos universitarios puede mejorar el proceso de enseñanza y aprendizaje por parte de todos los estudiantes.

Por ejemplo, el trabajo llevado a cabo por Spooner et al. (2007), muestra cómo la formación del profesorado sobre técnicas para desarrollar currículos basados en el diseño universal para el aprendizaje, incluso cuando las acciones formativas son muy breves,

Tabla 3

Resumen de las respuestas de estudiantes con discapacidad sobre medidas que favorecen la atención a la diversidad en el aula

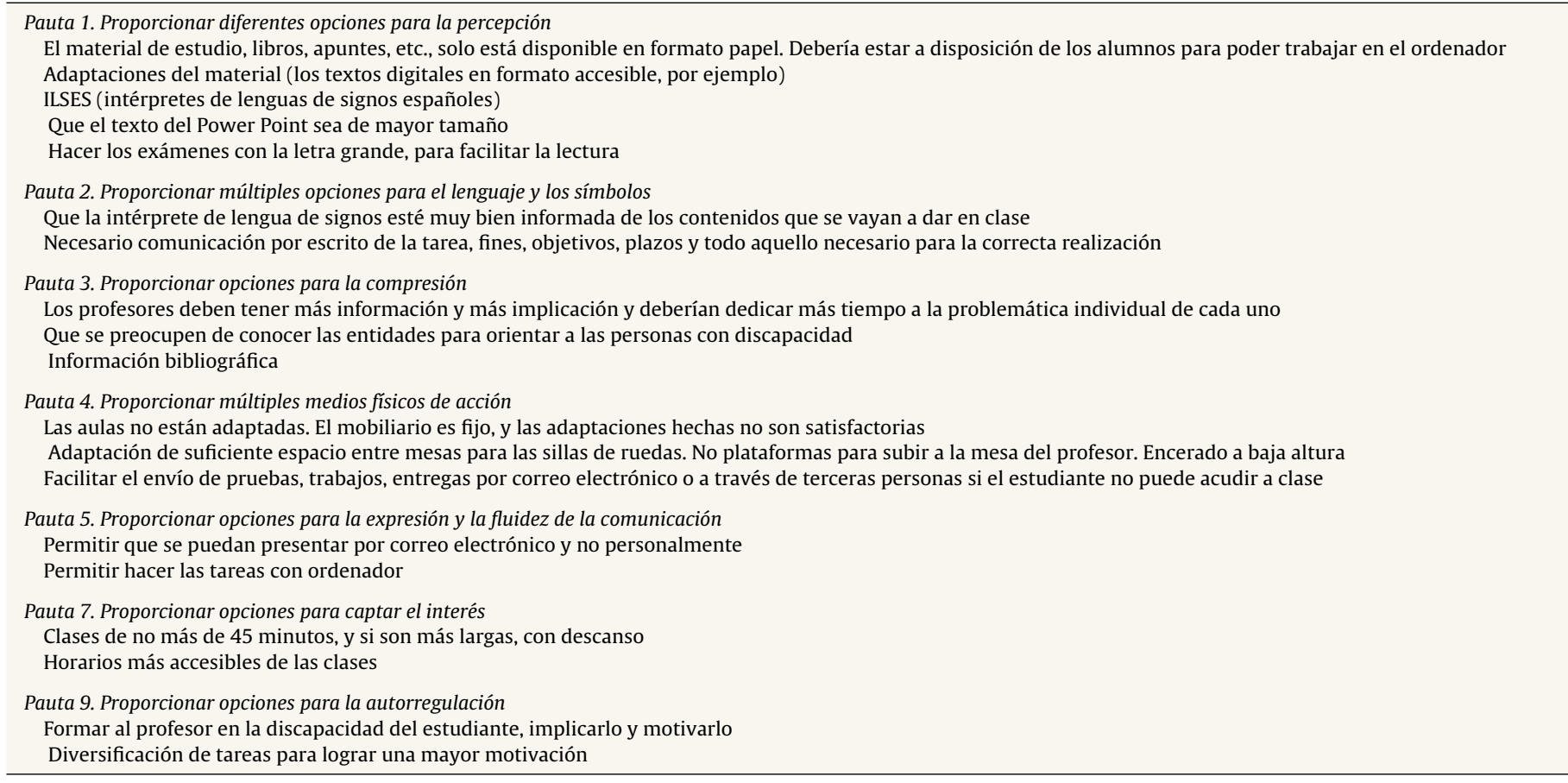


favorece el desarrollo de estrategias inclusivas en la creación de contenidos de aprendizaje haciéndolos más accesibles para todos los estudiantes. Spooner describe la realización de un seminario de una hora de duración en el que se presentaban los principios del DUA y unas pautas básicas de cómo implementarlo en el currículo a un grupo de profesores de educación básica y de educación especial. Los resultados de la evaluación del impacto del seminario mostraron que los grupos donde se había realizado la formación fueron capaces de crear unidades didácticas más inclusivas.

Igualmente relevante es el trabajo de Schelly, Davies y Spooner (2011), que pusieron en marcha un programa de formación a docentes en aspectos relacionados con el DUA, incluyendo la accesibilidad ofimática en la presentación de contenidos en las asignaturas, y logrando una mejora de las percepciones de los estudiantes en actuaciones relacionadas con el diseño universal. Los autores concluyen que el entrenamiento de los profesionales de la educación siguiendo las estrategias de implementación del DUA es percibida por los estudiantes como algo positivo, de manera que favorece que la educación en las aulas universitarias sea más inclusiva para todos.

En este contexto, desde el Instituto Universitario de Integración en la Comunidad de la Universidad de Salamanca se ha puesto en marcha una experiencia formativa para profesores universitarios titulada "diseño universal para el aprendizaje en la universidad». La selección de contenidos del curso se ha llevado a cabo a partir de la revisión de distintos talleres y cursos relacionados con la implementación de medidas de atención a estudiantes con discapacidad universitarios bajo los principios del diseño universal para el aprendizaje. Y en resumen, el curso está formado por distintos módulos que pretenden ofrecer una visión general respecto a las necesidades de los estudiantes con discapacidad y cómo afrontarlas mediante el diseño universal.

Así, el curso incluye un primer módulo sobre universidad y discapacidad en el que se presta especial atención a las experiencias en primera personas de estudiantes universitarios con discapacidad como medio para conocer las necesidades y la problemática de estos estudiantes. El segundo módulo introduce la temática de la accesibilidad de los contenidos educativos (web, software, contenidos, etc.) para ilustrar cómo una dificultad concreta, como la accesibilidad a los contenidos, puede ser superada si se diseña pensando en todos los usuarios. Esto conduce a un tercer módulo dedicado a introducir el paradigma del diseño universal y su aplicación concreta al ámbito de la educación. Posteriormente se introducen los principios y pautas del diseño universal para el aprendizaje, ofreciendo ejemplos concretos de su aplicación. Y además se llevan a cabo tutoriales sobre accesibilidad Web en el contexto de la plataforma moodle y sobre textos alternativos y documentos de ofimática accesibles.

El curso se ha integrado en el programa de formación continua para docentes de la Universidad de Salamanca del Instituto de Ciencias de la Educación y hasta ahora se han celebrado dos ediciones en las que han participado 40 profesores de todas las ramas de conocimiento. En las dos ediciones realizadas el curso se ha desarrollado en formato presencial de dos sesiones de 4 horas y con el apoyo de una plataforma virtual de aprendizaje con contenidos adicionales. Aunque no se ha realizado una evaluación exhaustiva de los resultados del curso, las evaluaciones disponibles muestran que los asistentes, en su mayoría, consideran que su participación en la actividad formativa les permite renovar su práctica como docentes y lo consideran una actividad importante dentro de su formación.

Para concluir este apartado no queremos dejar de destacar que la formación sobre los principios del diseño universal para profesionales que han de diseñar entornos, productos o servicios para personas con necesidades diversas es de vital importancia no solo para profesores universitarios y en relación a su labor educativa. De hecho, la propia legislación universitaria que regula los nuevos títulos de grado, ya desde su preámbulo, expresa que «se debe tener en cuenta que la formación en cualquier actividad profesional debe contribuir al conocimiento y desarrollo... de accesibilidad universal y diseño para todos». Y también especifica que «en los planes de estudios que proceda, se deberán incluir enseñanzas relacionadas con los derechos fundamentales y de igualdad de oportunidades entre hombres y mujeres, con los principios de igualdad de oportunidades y accesibilidad universal de las personas con discapacidad y con los valores propios de una cultura de la paz y de valores democráticos» (Real Decreto 1393/2007).

Aunque algunas universidades ya han incluido en los currículos de muchas de sus titulaciones contenidos sobre diseño universal, algunos estudios recientes muestran que aún queda mucho trabajo por hacer (Díez et al., 2011; Iglesias, Saravia y Lloredo, 2011; Sánchez, 2013). No obstante, además de acciones formativas explícitas, un buen camino para que los futuros profesionales asuman los principios del diseño universal es que ellos mismos puedan experimentar sus beneficios durante el desarrollo de las actividades de sus estudios universitarios. Y en este contexto, la formación del profesorado se configura como una de las estrategias clave para que el diseño pensado para todos se integre de manera natural en el sistema educativo.

\section{Conclusiones}

La atención a la diversidad es uno de los requerimientos que la actual legislación hace a las instituciones educativas. Por lo tanto, las universidades tienen la obligación de atender a todos los estudiantes, independientemente de cuáles sean su características o condiciones personales. Además, esta atención debe ser de calidad y ofrecer las mismas oportunidades en todos los aspectos de la vida universitaria. En la mayoría de los casos esta atención está cubierta gracias a los servicios de atención a la discapacidad, que son los encargados de velar por los estudiantes con distintas capacidades en su paso por la universidad. Sin embargo, en el plano académico deben ser los profesores los que den soporte a estas necesidades que pueden presentar los estudiantes. Y, es aquí, donde reside la necesidad de que los profesores desarrollen competencias basadas en la atención a la diversidad, para así poder ofrecer una formación adecuada a las necesidades de sus estudiantes.

Como medio para atender a la diversidad en las aulas universitarias, este trabajo ha defendido la aplicación del paradigma del diseño universal a la educación como una estrategia válida y con cierto apoyo empírico. Especialmente, hemos considerado el diseño universal para el aprendizaje como uno de los principales paradigmas a tener en cuenta. En este sentido, y con el objetivo de ilustrar cómo la aplicación del DUA en la educación universitaria beneficia a todos los estudiantes, hemos presentado un estudio con estudiantes con discapacidad cuyos resultados han mostrado aquellas demandas expresadas por estos estudiantes que tienen relación directa con las premisas en las que se fundamenta la aplicación del DUA. Por ello, dichas demandas podrían reducirse, o incluso, eliminarse si el diseño universal para el aprendizaje fuera aplicado desde los momentos iniciales del diseño curricular académico.

Finalmente, defendemos la necesidad de formar a los profesores universitarios en la aplicación del paradigma del diseño universal para el aprendizaje y sus ventajas a la hora de proporcionar las mismas oportunidades para todos los estudiantes, independientemente de sus características individuales. Sin duda, de este modo, se podrá mejorar la atención a la diversidad en la universidad.

\section{Conflicto de intereses}

Los autores declaran no tener ningún conflicto de intereses. 


\section{Referencias bibliográficas}

Bryson, J. (2003). Universal instructional design in postsecondary setting [consultado 1 Feb 2013]. Disponible en: www.mohawkcollege.ca/Assets/ StudentDev/.../UID+Manual.pdf

CAST. (2011). Universal design for learning guidelines version 2.0. Wakefield, MA [consultado 10 Feb 2012]. Disponible en: http://www.udlcenter.org/ sites/udlcenter.org/files/UDL_Guidelines_Version_2.0_(Final)_3.doc

Darr, A. y Jones, R. (2008). The contribution of universal design to learning and teaching excellence. In Universal design in higher education: From principles to practice. Cambridge MA: Harvard Education Press.

Díez, E., Alonso, A., Verdugo, M. A., Campo, I., Sancho, I., Sánchez, S. ... Moral, E. (2011). Espacio Europeo de Educación Superior: estándares e indicadores de buenas prácticas para la atención a estudiantes universitarios con discapacidad (Instituto de Integración en la Comunidad, Universidad de Salamanca.). Salamanca: Instituto de Integración en la Comunidad, Universidad de Salamanca.

Díez, E., Campo, M. y Sánchez, S. (2011). Estudio y análisis de la aplicación del R.D. 1393/2007, de 29 de octubre, por el que se establece la ordenación de las enseñanzas universitarias oficiales, en cuanto a la implantación de materias relacionadas con la accesibilidad y diseño para todos en los títulos de grado del ámbito de la ingeniería informática. Informe técnico no publicado. Instituto Universitario de Integración en la Comunidad, Universidad de Salamanca, Salamanca.

Fundación Universia (2013). Guía de atención a la discapacidad en la universidad. Madrid [consultado 1 Mar 2014]. Disponible en: http://www. fundacionuniversia.net/programas/informacion/guias/detalleProgramas-2161. html

Hill, J. (1996). Speaking out: perceptions of students with disabilities regarding adequacy of services and willingness of faculty to make accommodations. Journa of Postsecondary Education and Disability, 12, 22-43.

Hitchcock, C. y Stahl, S. (2003). Assistive technology, universal design, universal design for learning: Improved learning opportunities. Journal of Special Educational Technology, 19(4), 45-52.

Iglesias, A., Saraiva, G., y Lloredo, L. (2011). Informe sobre la presencia de la accesibilidad universal en las enseñanzas universitarias: Sobre la aplicación del artículo 3.5 del Real Decreto 1393/2007, de 29 de octubre, por el que se establece la Ordenación de la Enseñanzas Universitarias Oficiales (No. 17). HURI-AGE. Madrid Clínica Jurídica "La enseñanza de los derechos fundamentales en la universidad española» del Instituto de derechos humanos Bartolomé de las Casas de la Universidad Carlos III de Madrid [consultado 1 Mar 2013]. Disponible en http://www.tiempodelosderechos.es/docs/oct11/informe17.pdf

Ley $51 / 2003$, de 2 de diciembre, de igualdad de oportunidades, no discriminación y accesibilidad universal de las personas con discapacidad persona (2 de diciembre).

Ley Orgánica 4/2007, de 12 de abril, por la que se modifica la Ley Orgánica 6/2001, de 21 de diciembre, de Universidades. (2007).

McGuire, J., Scott, S. y Shaw, S. (2006). Universal design and its aplications in educational environments. Remedial and special education, 27(3), 166-175.

Meyer, A., y Rose, D. (2005). The universally designed classroom: accessible curriculum and digital technologies (Eds. Rose, D., Meyer, A. y Hitchcock, C.). Cambridge, MA: Harvard Education Press.
Meyer, A., y Rose, D. (2006). A practical reader in universal design for learning (Eds. Rose, D. y Meyer, A.). Cambridge, MA: Harvard Education Press.

Meyer, A. y Rose, D. (2009). A policy reader in universal design for learning. Cambridge, MA: Harvard Education Press.

Moral, E.(2009). Estudio exploratorio sobre la percepción subjetiva de la atención a la discapacidad por parte de la comunidad universitaria. Las adaptaciones curriculares como medida integradora. Trabajo de Grado no publicado. Universidad de Salamanca, Instituto Universitario de Integración en la Comunidad, Salamanca.

National Center for Universal Design for Learning (2012). UDL Guideline - Version 2.0 [consultado 1 Abr 2012]. Disponible en: http://www.udlcenter org/aboutudl/udlguidelines

Peralta, A. (2007). Libro blanco sobre universidad y discapacidad. Madrid: Real Patronato sobre Discapacidad.

Real Decreto 1393/2007, de 29 de octubre, por el que se establece la ordenación de las enseñanzas universitarias oficiales. (2007).

Real Decreto 1791/2010, de 30 de diciembre, por el que se aprueba el Estatuto del Estudiante Universitario.

Real Decreto 1892/2008, de 14 de noviembre, por el que se regulan las condiciones para el acceso a las enseñanzas universitarias oficiales.

Rose, D., Harbour, W., Johnston, C., Daley, S. y Abarbanell, L. (2006). Universal design for learning in postsecondary education: reflections on principles and their application. Journal of Postsecondary Education and Disability, 19(2), 135-151.

Rose, D. y Meyer, A. (2000). Universal design for learning. Journal of Special Education Technology, 15, 67-70

Rose, D. y Meyer, A. (2002). Teaching every student in the digital age: universal design for learning. VA: ASCD.

Ruiz, R., Solé, L., Echeita, G., Sala, I. y Datsira, M. (2012). El principio del Universa Design. Concepto y desarrollos en la enseñanza superior. Revista de Educación. doi:10-4438/1988-592X-RE-2010-359-100.

Sánchez, S., Díez, E., Verdugo, M.A., Iglesias, A., y Calvo, I. (2011). Atención a la diversidad en las titulaciones adaptadas al RD 1393/2007: Adaptación de una herramienta Web de autoevaluación curricular basada en los principios del diseño universal para el aprendizaje. En Jornadas de Innovación Docente en la Universidad de Salamanca (Universidad de Salamanca (España). Vicerrectorado de Docencia., pp. 148-155). Salamanca. Disponible en: http://gredos.usal.es/jspui/handle/10366/112888

Sánchez, S. (2013). Aplicación del paradigma del diseño universal en la educación universitaria española: implantación de estudios y percepción sobre inclusión de medidas curriculares. Tesis Doctoral no publicada. Universidad de Salamanca, Instituto Universitario de Integración en la Comunidad, Salamanca.

Schelly, C., Davies, P. y Spooner, C. (2011). Student perceptions of faculty implementation of universal design for learning. Journal of Postsecondary Education and Disability, 24(1), 17-30.

Scott, S., McGuire, J. y Shaw, S. (2001). Principles of Universal Design for Instruction. Storrs, CT.

Spooner, F., Baker, J., Harris, A., Ahlgrim-Delzell, L. y Browder, D. (2007). Effects of training in universal design for learning on lesson plan development. Remedial and special education, 28(2), 108-116.

Yuval, L., Procter, E., Korabik, K., y Palmer, J. (2004). Evaluation report on the universal instructional design at the University of Guelph. University of Guelph: University of Guelph. Disponible en: http://www.coles.uoguelph.ca/ TSS/instructional_design/highlights.aspx 